Pacific Journal of Mathematics

DEFINITE AND SEMIDEFINITE MATRICES IN A REAL 


\section{DEFINITE AND SEMIDEFINITE MATRICES IN A REAL SYMMETRIC MATRIX PENCIL}

\section{FRANK UHLIG}

Pencils that contain a definite matrix ( $d$-pencils) have been characterized in several ways. Here $d$-pencils will be characterized by the property of the set $L=\left\{\left(a_{i}, b_{i}\right)\right\} \subseteq R^{2}$ if $S$ and $T$ are simultaneously congruent to $\operatorname{diag}\left(a_{i}\right)$ and $\operatorname{diag}\left(b_{i}\right)$, respectively. This way one can describe all definite and semidefinite matrices in a $d$-pencil. Similarly one can characterize all pencils that contain semidefinite but no definite matrices (s.d. pencils). The explicit condition on $L$ for $d$-pencils is then used to reprove the theorem that two real symmetric matrices generate a $d$-pencil iff their associated quadratic forms do not vanish simultaneously.

Definition 1. If $S$ is symmetric we define $Q_{S}=\left\{x \in \boldsymbol{R}^{n} \mid x^{\prime} S x=0\right\}$.

Definition 2. For real symmetric (r.s.) matrices $S$ and $T$ one defines the pencil $P(S, T)=\{a S+b T \mid a, b \in \boldsymbol{R}\}$.

Definition 3. (a) $P(S, T)$ is called $d$-pencil if $P(S, T)$ contains a definite matrix.

(b) $P(S, T)$ is called s.d. pencil if $P(S, T)$ contains a nonzero semidefinite, but no definite matrix.

The following theorem is classical:

THEOREM 0.1. If $P(S, T)$ is a d-pencil then $S$ and $T$ can be diagonalized simultaneously by a real congruence transformation.

The question whether a given pencil of r.s. matrices contains a positive definite matrix was treated in chronological order by Finsler [6], Albert [1], Reid [12], Hestenes and McShane [9], Dines [5], Calabi [4], Taussky [13], Hestenes [8], Theorem 3, and Berman [3].

Their main results are the following:

THEOREM 0.2. Let $S$ and $T$ be r.s. $n \times n$ matrices. If $n \geqq 3$, then the following are equivalent:

(i ) $P(S, T)$ is a d-pencil,

(ii) $Q_{S} \cap Q_{T}=\{0\}$, and

(iii) trace $Y S=$ trace $Y T=0$ for $Y$ positive semidefinite implies $Y=0$.

The equivalence of (i) and (ii) was proved by Calabi [4], while Berman [3] showed the equivalence of (i) and (iii). 
Before Calabi [4] only condition

$$
x^{\prime} S x=0 \quad \text { implies } \quad x^{\prime} T x>0
$$

was generally used instead of (ii). And thus only the fact that (ii') implies (i) was proved.

In view of Theorem 0.1 , to characterize $d$-pencils means to characterize the sets $L=\left\{\left(a_{i}, b_{i}\right) \mid i=1, \cdots, n\right\} \subseteq \boldsymbol{R}^{2}$ for which there exist $\lambda, \mu \in \boldsymbol{R}$ with $\lambda a_{i}+\mu b_{i}>0$ for all $i$.

TheOREM 1.1. $P(S, T)$ is a d-pencil iff $S$ and $T$ can be simultaneously diagonalized by a real congruence transformation $X: X^{\prime} S X=$ $\operatorname{diag}\left(a_{i}\right), X^{\prime} T X=\operatorname{diag}\left(b_{i}\right)$, and in case that there are indices $i, j$ with $a_{i} a_{j}<0$ we have

(a) $\max _{a_{i}>0} \frac{b_{i}}{a_{i}}<\max _{a_{i}<0} \frac{b_{1}}{a_{i}}$ and $b_{i}<0$ whenever $a_{i}=0$, or

(b) $\min _{a_{i}>0} \frac{b_{i}}{a_{i}}>\min _{a_{i}<0} \frac{b_{i}}{a_{i}}$ and $b_{i}>0$ whenever $a_{i}=0$, while in case that all $a_{i}$ have one sign we have

(c) either $b_{i}<0$ whenever $a_{i}=0$ or $b_{i}>0$ whenever $a_{i}=0$.

Proof. If $P(S, T)$ is a $d$-pencil, then by Theorem 0.1 the matrices $S$ and $T$ can be diagonalized simultaneously by a real congruence transformation: $X^{\prime} S X=\operatorname{diag}\left(a_{i}\right), X^{\prime} T X=\operatorname{diag}\left(b_{i}\right)$. Furthermore, there exist $\lambda, \mu \in \boldsymbol{R}$ s.t. $\lambda S+\mu T=X^{\prime} \operatorname{diag}\left(\lambda a_{i}+\mu b_{i}\right) X$ is positive definite.

Hence the set $L=\left\{\left(a_{i}, b_{i}\right) \mid i=1, \cdots, n\right\} \subseteq \boldsymbol{R}^{2}$ must lie in an open half plane of $\boldsymbol{R}^{2}$ and it follows that either (a) or (b) or (c) must hold for $L$.

Conversely if $S$ and $T$ are simultaneously congruent to $\operatorname{diag}\left(a_{i}\right)$ and $\operatorname{diag}\left(b_{i}\right)$ then in case of (a) all points $\left(a_{i}, b_{i}\right) \in \boldsymbol{R}^{2}$ lie "below" any line $L_{\alpha}$ thru zero that has slope $\max _{a_{i}>0} b_{i} / a_{i}<\alpha<\max _{a_{i}<0} b_{i} / a_{i}$. In case of (b), they all lie "above" any such line $L_{\alpha}$ with $\min _{a_{i}>0} b_{i} / a_{i}>$ $\alpha>\min _{a_{i}<0} b_{i} / a_{i}$ and in case of (c) they all lie to the "right" or to the "left". of any line $L_{\alpha}$ where either

$$
\max _{a_{i}>0} \frac{b_{i}}{a_{i}}<\alpha<\infty \quad \text { or }-\infty<\alpha<\min _{a_{i}>0} \frac{b_{i}}{a_{i}} .
$$

Thus the set $L$ lies in an open half plane and hence there exist $\lambda, \mu \in \boldsymbol{R}$ s.t. $\lambda a_{i}+\mu b_{i}>0$ for all $i$. Thus $P(S, T)$ is a $d$-pencil. 
Next, if $P(S, T)$ is a $d$-pencil we will explicitly describe the set $M^{+}=\{(\lambda, \mu) \mid \lambda S+\mu T$ pos. def. $\} \subseteq R^{2}$ and determine the positive semidefinite matrices in $P(S, T)$. The sets $M^{+}$and its closure $M=$ $\{(\lambda, \mu) \mid \lambda S+\mu T$ pos. semidef. $\}$ have been previously characterized as convex cones and this fact together with properties of quadratic forms have been used to prove the equivalence of (i) and (ii) in Theorem 0.2 (Hestenes [8]). Hestenes [8] moreover treats related questions for positively elliptic pairs of quadratic forms in Hilbert space.

THEOREM 1.2. Let $P(S, T)$ be a d-pencil and let $S, T$ be simultaneously congruent to $\operatorname{diag}\left(a_{i}\right)$ and $\operatorname{diag}\left(b_{i}\right)$.

Then with (a), (b), and (c) from Theorem 1.1 we have

(1) $\lambda S+\mu T$ is positive definite iff

in case of (a) $-\left(\max _{a_{i}>0} \frac{b_{i}}{a_{i}}\right)^{-1}<\frac{\lambda}{\mu}<-\left(\max _{a_{i}<0} \frac{b_{i}}{a_{i}}\right)^{-1}$ and

in case of (b) $-\left(\min _{a_{i}>0} \frac{b_{i}}{a_{i}}\right)^{-1}>\frac{\lambda}{\mu}>-\left(\min _{a_{i}<0} \frac{b_{i}}{a_{i}}\right)^{-1}$ while

in case of (c) if all $a_{i}$ are nonnegative

$$
-\left(\max _{a_{i}>0} \frac{b_{i}}{a_{i}}\right)^{-1}<\frac{\lambda}{\mu}<0 \text { if } b_{i}<0 \text { whenever } a_{i}=0
$$

and

$$
0<\frac{\lambda}{\mu}<-\left(\min _{a_{i}>0} \frac{b_{i}}{a_{i}}\right)^{-1} \quad \text { if } b_{i}>0 \text { whenever } a_{i}=0
$$

and, if all $a_{i} \leqq 0$, we have

$$
-\left(\max _{a_{i}<0} \frac{b_{i}}{a_{i}}\right)^{-1}>\frac{\lambda}{\mu}>0 \quad \text { if } b_{i}<0 \text { whenever } a_{i}=0
$$

while

$$
0>\frac{\lambda}{\mu}>-\left(\min _{a_{i}<0} \frac{b_{i}}{a_{i}}\right)^{-1} \quad \text { if } b_{i}>0 \text { whenever } a_{i}=0 .
$$

(2) $\lambda S+\lambda T$ is positive semidefinite and not definite iff in each case respectively, $\lambda / \mu$ is equal to either one of the endpoints of the intervals under (1).

Here we set

$$
(0 / a)^{-1}=\left\{\begin{aligned}
\infty & \text { if } a>0 \\
-\infty & \text { if } a<0
\end{aligned}\right.
$$

Proof. The proof follows from Theorem 1.1 and by the geometry 
of $\boldsymbol{R}^{2}$. Concerning (2) one can say that if $\lambda / \mu$ is an endpoint of an appropriate interval in (1), then $\lambda S+\mu T$ is positive semidefinite of rank $n-r \leqq n$ iff $r=\left|\left\{j \mid b_{i} / a_{j}=\lambda / \mu\right\}\right|$.

As corollaries we observe that

COROLlaRY 1. If in Theorem 1.1 the strict reverse inequalities hold in (a) and (b), or (c), respectively, then $\mu S+\lambda T$ is indefinite for any $(\lambda, \mu) \neq(0,0)$. And then $\mu S+\lambda T$ has at least rank 2 for any $(\lambda, \mu) \neq(0,0)$.

Before characterizing s.d. pencil we will quote a simplified version of the canonical pair form theorem for nonsingular pairs of r.s. matrices.

DEFINITION 4. A pair of r.s. matrices $S, T$ is called nonsingular, if $S$ is nonsingular.

THEOREM 1.3. Let $S$ and $T$ be a nonsingular pair of r.s. matrices. Let $S^{-1} T$ have real Jordan normal form diag $\left(J_{1}, \cdots, J_{m}\right)$. Then $S$ and $T$ are simultaneously congruent by a real congruence transformation to diag $\left(\varepsilon_{i} E_{i}\right)$ and $\operatorname{diag}\left(\varepsilon_{i} E_{i} J_{i}\right)$ respectively, where $\varepsilon_{i}= \pm 1$ and $E_{i}$ denotes

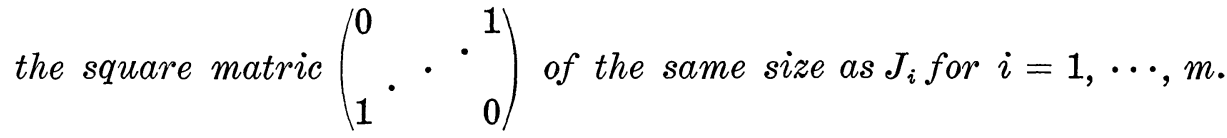

The relevant notation has recently been described in Uhlig [15]. There it was shown that the canonical pair form just described is a finest simultaneous block diagonalization.

Note that if $J=\left(\begin{array}{llll}\lambda & 1 & & \\ & \cdot & & \\ & \cdot & & \\ 0 & & \lambda_{\lambda} & 1\end{array}\right)$ is a Jordan block for $\lambda \in \boldsymbol{R}$ then



$$
a E+b E J=\left(\begin{array}{llrr}
0 & & a+b \lambda \\
& \cdot & \cdot & b \\
a+b \lambda & b & 0
\end{array}\right) .
$$

Hence $a E+b E J$ is indefinite for all $(a, b) \neq(0,0)$. The same holds for any $J$ corresponding to an eigenvalue $\lambda=\alpha+\beta i \notin \boldsymbol{R}$. With this in mind we can characterize s.d. pencils:

THEOREM 1.4. Let $S, T$ be a nonsingular pair of r.s. matrices of dimension greater than 2. 
Then $P(S, T)$ is a s.d. pencil iff either $S$ and $T$ are simultaneously congruent to $\operatorname{diag}\left(a_{i}\right)$ and $\operatorname{diag}\left(b_{i}\right)$ with $b_{i} / a_{i} \neq b_{j} / a_{j}$ for at least one pair of indices $(i, j)$ and we have

$$
\text { (a') } \max _{a_{i}>0} \frac{b_{i}}{a_{i}}=\max _{a_{i}<0} \frac{b_{i}}{a_{i}} \text { or } \quad\left(b^{\prime}\right) \min _{a_{i}>0} \frac{b_{i}}{a_{i}}=\min _{a_{i}<0} \frac{b_{i}}{a_{i}},
$$

or $S$ and $T$ are simultaneously congruent to

$$
\operatorname{diag}\left(\varepsilon E, \cdots, \varepsilon E, \varepsilon_{k+1}, \cdots, \varepsilon_{j}, a_{j+1}, \cdots, a_{n}\right)
$$

and

$$
\operatorname{diag}\left(\varepsilon E J, \cdots, \varepsilon E J, \varepsilon_{k+1} \alpha, \cdots, \varepsilon_{j} \alpha, b_{j+1}, \cdots, b_{n}\right) \text {, }
$$

where $J=\left(\begin{array}{cc}\alpha & 1 \\ 0 & \alpha\end{array}\right), \quad E=\left(\begin{array}{ll}0 & 1 \\ 1 & 0\end{array}\right) ; \quad \varepsilon, \varepsilon_{i}= \pm 1, \quad \alpha \in R, \quad b_{l} \neq \alpha a_{l} \quad$ and $\varepsilon, b_{l}-\alpha a_{l}$ all have the same sign for $l=j+1, \cdots, n$.

Proof. We recall that $P(S, T)$ is a s.d. pencil if there is a semidefinite but no definite matrix in $P(S, T)$.

If $S$ and $T$ can be diagonalized simultaneously to yield $\operatorname{diag}\left(a_{i}\right)$ and $\operatorname{diag}\left(b_{i}\right)$, then $P(S, T)$ is a s.d. pencil iff $L=\left\{\left(a_{i}, b_{i}\right)\right\} \subseteq \boldsymbol{R}^{2}$ lies in a closed half plane, but in no open half plane of $\boldsymbol{R}^{2}$, nor on a line thru 0; which is the case iff $\left(a^{\prime}\right)$ or $\left(b^{\prime}\right)$ holds. (Note that since $S$ is nonsingular we have $a_{i} \neq 0$ for all $i$.)

If $P(S, T)$ is a s.d. pencil and $S$ and $T$ cannot be simultaneously diagonalized, then the real Jordan normal form $J_{0}$ of $S^{-1} T$ cannot contain a Jordan block of dimension greater than two for a real eigenvalue, nor can $S^{-1} T$ have complex roots as we have observed in the sequel of Theorem 1.3. But for $J=\left(\begin{array}{cc}\alpha & 1 \\ 0 & \alpha\end{array}\right)$ and $E=\left(\begin{array}{ll}0 & 1 \\ 1 & 0\end{array}\right)$ we have $\lambda E+\mu E J=$ $\left(\begin{array}{cc}0 & \lambda+\mu \alpha \\ \lambda+\mu \alpha & \mu\end{array}\right)$ is semidefinite iff $\lambda+\mu \alpha=0$. Hence if there are several 2-dimensional blocks in $J_{0}$ they must all correspond to the same eigenvalue $\alpha \in \boldsymbol{R}$ and they all must carry the same sign $\varepsilon$. The signs of the one-dimensional blocks corresponding to the same eigenvalue $\alpha$ cannot be specified since $\varepsilon_{i}(\lambda+\mu \alpha)=0$ independent of $\varepsilon_{i}= \pm 1$, but for one-dimensional blocks in $J_{0}$ not corresponding to $\alpha$, i.e., if $b_{l} \neq \alpha a_{l}$, then we must have that $\varepsilon \mu$ and $\lambda a_{l}+\mu b_{l}=\mu\left(b_{l}-\alpha a_{l}\right)$ all have the same $\operatorname{sign}$ for $l=j+1, \cdots, n$. If $\mu=0$ we conclude that if $\lambda E+$ $\mu E J=\lambda E$ is semidefinite then $\lambda=0$. Thus $\mu$ cannot be zero. And the theorem is proved, since the converse is obvious in this case.

Finally we will apply Theorem 1.1 to give a new elementary proof of the equivalence of (i) and (ii) in Theorem 0.2 . 
For this we need a theorem of Greub and Milnor [7, p. 256]:

THEOREM 2.1. Let $S, T$ be r.s. matrices of dimension greater than 2. If $Q_{S} \cap Q_{T}=\{0\}$, then $S$ and $T$ can be diagonalized simultaneously by a real congruence transformation.

This Theorem was also proved by Majindar [11], Kraljevic [10], Wonenburger [16], Au-Yeung [1], and the author [14].

Proof. [(i) and (ii) of Theorem 0.2 are equivalent.] That (i) implies (ii) is obvious since for definite matrices $U$ we have $Q_{U}=\{0\}$ and clearly for any $U \in P(S, T)$ we have $Q_{U} \supseteqq Q_{S} \cap Q_{T}$.

If (ii) holds, then by Theorem 2.1 the matrices $S$ and $T$ are simultaneously congruent to $D_{1}=\operatorname{diag}\left(a_{i}\right)$ and $D_{2}=\operatorname{diag}\left(b_{i}\right)$. We are going to show that

$$
\sum a_{i} x_{i}^{2}=\sum b_{i} x_{i}^{2}=0 \quad \text { implies } x_{i}=0 \text { for all } i
$$

cannot hold unless either (a) or (b) or (c) of Theorem 1.1 is the case.

In the case that, say, all $a_{i} \geqq 0$ and (c) is violated, then we have $a_{i}=a_{j}=0$ for $i \neq j$, while $b_{i} \geqq 0$ and $b_{j} \leqq 0$. With $e_{i}$ denoting the $i$ th unit vector we then have

$$
0 \neq x=e_{i}+\sqrt{\frac{b_{i}}{-b_{j}}} \cdot e_{j} \in Q_{D_{1}} \cap Q_{D_{2}} \text { if } b_{j} \neq 0,
$$

while else $e_{j} \in Q_{D_{1}} \cap Q_{D_{2}}$, hence $Q_{S} \cap Q_{T} \neq\{0\}$ as well.

In the case that there are positive as well as negative $a_{i}$ and if both

$$
\left[\max _{a_{i}>0} \frac{b_{i}}{a_{i}} \geqq \max _{a_{i}<0} \frac{b_{i}}{a_{i}} \text { or } b_{i} \geqq 0 \text { for some } a_{i}=0\right]
$$

and

$$
\left[\min _{a_{i}>0} \frac{b_{i}}{a_{i}} \leqq \min _{a_{i}<0} \frac{b_{i}}{a_{i}} \text { or } b_{i}<0 \text { for some } a_{i}=0\right]
$$

hold, i.e., if both (a) and (b) of Theorem 1.1 are violated, then we will show that $Q=Q_{D_{1}} \cap Q_{D_{2}} \neq\{0\}$ and then the equivalence of (i) and (ii) will be proved.

We now go into subcases:

If both $b_{i} \geqq 0$ and $b_{j} \leqq 0$ for $a_{i}=a_{j}=0$, then $Q_{D_{1}} \cap Q_{D_{2}} \neq\{0\}$ as we have just seen.

If both $\max _{a_{i}>0} b_{i} / a_{i} \geqq \max _{a_{i}<0} b_{i} / a_{i}$ and $b_{i} \leqq 0$ for some $a_{i}=0$ hold, then we assume without loss of generality that the indices in question are $1,2,3$, respectively, i.e., $b_{1} / a_{1} \geqq b_{2} / a_{2}$ where $a_{1}>0, a_{2}<0$, and $a_{3}=0$, while $b_{3} \leqq 0$. 
If $b_{3}=0$, then $e_{3} \in Q$. If $b_{1} / a_{1}=b_{2} / a_{2}$, then $e_{1}+\left(\sqrt{-a_{1} / a_{2}}\right) e_{2} \in Q$. Otherwise with $x=\left(1, \sqrt{-a_{1} / a_{2}}, \alpha, 0, \cdots, 0\right)$ we get

$$
\sum a_{i} x_{i}^{2}=a_{1}-a_{1} \frac{a_{2}}{a_{2}}=0 \text { independent of } \alpha,
$$

and

$$
\sum b_{i} x_{i}^{2}=b_{1}-\frac{b_{2} a_{1}}{a_{2}}+b_{3} \alpha^{2}
$$

Now by assumption $b_{1}-\left(a_{1} / a_{2}\right) b_{2}>0$ and $b_{3}<0$, hence there is a real $\alpha$ s.t. $\sum b_{i} x_{i}^{2}=0$ as well, and consequently $Q \neq\{0\}$. If both $\min _{a_{i}>0} b_{i} / a_{i} \leqq \min _{a_{i}<0} b_{i} / a_{i}$ and $b_{i} \geqq 0$ for some $a_{i}=0$ hold, then a similar argument applies.

Finally assume both

$$
\left(^{*}\right) \max _{a_{i}>0} \frac{b_{i}}{a_{i}} \geqq \max _{a_{i}<0} \frac{b_{i}}{a_{i}} \text { and } \min _{a_{i}>0} \frac{b_{i}}{a_{i}} \leqq \min \frac{b_{i}}{a_{i}} \text { hold . }
$$

If in (*) there is an equal sign, say $b_{1} / a_{1}=b_{2} / a_{2}$ with $a_{1}>0, a_{2}<0$, then as above $e_{1}+\left(\sqrt{-a_{1} / a_{2}}\right) e_{2} \in Q$. Thus the only case to remain to be proved is if both inequalities in $\left(^{*}\right)$ are strict. For $n \geqq 4$ assume that the indices in question are $1,2,3,4$; i.e., $b_{1} / a_{1}>b_{2} / a_{2}$ with $a_{1}>0, a_{2}<0$ and $b_{3} / a_{3}<b_{4} / a_{4}$ with $a_{3}>0, a_{4}<0$. Then with $x=\left(1,\left(\sqrt{-a_{1} / a_{2}}\right), \alpha\right.$, $\left.\left(\sqrt{-a_{3} / a_{4}}\right) \alpha, 0, \cdots, 0\right)$ we have

$$
\sum a_{i} x_{i}^{2}=a_{1}-a_{1}+\alpha^{2}\left(a_{3}-a_{3}\right)=0 \text { independently of } \alpha,
$$

and

$$
\sum b_{i} x_{i}^{2}=b_{1}-\frac{a_{1}}{a_{2}} b_{2}+\alpha^{2}\left(b_{3}-b_{4} \frac{a_{3}}{a_{4}}\right) .
$$

By assumption $b_{1}-\left(a_{1} / a_{2}\right) b_{2}>0$ and $b_{3}-b_{4}\left(a_{3} / a_{4}\right)<0$. Thus $\alpha \in \boldsymbol{R}$ can be chosen s.t. $\sum b_{i} x_{i}^{2}=0$.

For $n=3$ we may WLOG assume that $\min _{a_{i}>0} b_{i} / a_{i}=\max _{a_{i}>0} b_{i} / a_{i}=$ $b_{1} / a_{1}$. Then $\left(^{*}\right)$ reads like $b_{1} / a_{1}>b_{2} / a_{2}$ and $b_{1} / a_{1}<b_{3} / a_{3}$ with $a_{1}>0$; $a_{2}, a_{3}<0$. Then with $x=\left(1, \sqrt{-a_{1} \alpha / a_{2}}, \sqrt{-a_{1} \beta / a_{3}}\right)$ we have

$$
\sum a_{i} x_{i}^{2}=a_{1}-a_{1}(\alpha+\beta) \text { and } \sum b_{i} x_{i}^{2}=b_{1}-\frac{b_{2} a_{1}}{a_{2}} \alpha-\frac{b_{3} a_{1}}{a_{3}} \beta .
$$

Now the linear system in $\alpha$ and $\beta$ :

$$
\alpha+\beta=1 \quad \frac{b_{2} a_{1}}{a_{2}} \alpha+\frac{b_{3} a_{1}}{a_{3}} \beta=b_{1}
$$

can be solved for arbitrary $b_{1}$, since its determinant 


$$
a_{1}\left(\frac{b_{3}}{a_{3}}-\frac{b_{2}}{a_{2}}\right) \neq 0
$$

by assumption.

This completes the proof.

\section{REFERENCES}

1. A. A. Albert, A quadratic form problem in the calculus of variations, Bull. Amer. Math. Soc., 44 (1938), 250-253.

2. Y.-H. Au-Yeung, Some theorems on the real pencil and simultaneous diagonalization of two hermitian bilinear forms, Proc. Amer. Math. Soc., 23 (1969), 246-253.

3. A. Berman, Thesis, Northwestern University, Evanston, III., 1970.

4. E. Calabi, Linear systems of real quadratic forms, Proc. Amer. Math. Soc., 15 (1964), 844-846.

5. L. Dines, On the mapping of quadratic forms, Bull. Amer. Math. Soc., 47 (1941), 494-498.

6. P. Finsler, Über das Vorkommen definiter und semidefiniter Formen in Scharen quadratischer Formen, Commentarii Math. Helvetici, 9 (1937), 188-192.

7. W. Greub, Linear Algebra, Springer, Berlin, $3^{\text {rd }}$ edition, 1967.

8. M. Hestenes, Pairs of quadratic forms, Linear Algebra and Appl. 1 (1968), 397-407.

9. M. Hestenes and McShane, Quadratic forms and the calculus of variations, Trans. Amer. Math. Soc., 38 (1935), 145.

10. H. Kraljevic, Simultaneous diagonalization of two symmetric bilinear symmetric functionals, Glasnik Matematicki, 1 (1966), 57-63.

11. K. Majindar, On simultaneous hermitian congruence transformations of matrices, Amer. Math. Monthly, 70 (1963), 842-844.

12. W. Reid, A theorem on quadratic forms, Bull. Amer. Math. Soc., 44 (1938), 437.

13. O. Taussky, Positive Definite Matrices, in "Inequalities", O. Shisha, Ed., Academic Press, N.Y., 1967.

14. F. Uhlig, $A$ study of the canonical form of a pair of real symmetric matrices and applications to pencils and to pairs of quadratic forms, Thesis, California Institute of Technology, 1971-72.

15. - Simultaneous block diagonalization of two real symmetric matrices, Linear Algebra and Appl., 7 (1973), 281-289.

16. M. Wonenburger, Simultaneous diagonalization of symmetric bilinear forms, J. of Math. and Mech., 15 (1966), 617-622.

Received July 12, 1972.

UNIVERSITÄT WÜRZBURG 


\section{PACIFIC JOURNAL OF MATHEMATICS}

\section{EDITORS}

RICHARD ARENS (Managing Editor)

University of California

Los Angeles, California 90024

\section{R. A. Beaumont}

University of Washington

Seattle, Washington 98105

\section{J. DuGundJI*}

Department of Mathematics

University of Southern California

Los Angeles, California 90007

D. Gilbarg and J. Milgram

Stanford University

Stanford, California 94305

ASSOCIATE EDITORS
E. F. BECKENBACH
B. H. NeumanN
F. WOLF
K. YoSHIDA

\section{SUPPORTING INSTITUTIONS}

UNIVERSITY OF BRITISH COLUMBIA

CALIFORNIA INSTITUTE OF TECHNOLOGY

UNIVERSITY OF CALIFORNIA

MONTANA STATE UNIVERSITY

UNIVERSITY OF NEVADA

NEW MEXICO STATE UNIVERSITY

OREGON STATE UNIVERSITY

UNIVERSITY OF OREGON

OSAKA UNIVERSITY

\author{
UNIVERSITY OF SOUTHERN CALIFORNIA \\ STANFORD UNIVERSITY \\ UNIVERSITY OF TOKYO \\ UNIVERSITY OF UTAH \\ WASHINGTON STATE UNIVERSITY \\ UNIVERSITY OF WASHINGTON \\ AMERICAN MATHEMATICAL SOCIETY \\ NAVAL WEAPONS CENTER
}

The Supporting Institutions listed above contribute to the cost of publication of this Journal, but they are not owners or publishers and have no responsibility for its content or policies.

Mathematical papers intended for publication in the Pacific Journal of Mathematics should be in typed form or offset-reproduced, (not dittoed), double spaced with large margins. Underline Greek letters in red, German in green, and script in blue. The first paragraph or two must be capable of being used separately as a synopsis of the entire paper. Items of the bibliography should not be cited there unless absolutely necessary, in which case they must be identified by author and Journal, rather than by item number. Manuscripts, in duplicate if possible, may be sent to any one of the four editors. Please classify according to the scheme of Math. Rev. Index to Vol. 39. All other communications to the editors should be addressed to the managing editor, or Elaine Barth, University of California, Los Angeles, California, 90024.

50 reprints are provided free for each article; additional copies may be obtained at cost in multiples of 50 .

The Pacific Journal of Mathematics is issued monthly as of January 1966. Regular subscription rate: $\$ 48.00$ a year (6 Vols., 12 issues). Special rate: $\$ 24.00$ a year to individual members of supporting institutions.

Subscriptions, orders for back numbers, and changes of address should be sent to Pacific Journal of Mathematics, 103 Highland Boulevard, Berkeley, California, 94708.

\section{PUBLISHED BY PACIFIC JOURNAL OF MATHEMATICS, A NON-PROFIT CORPORATION}

Printed at Kokusai Bunken Insatsusha (International Academic Printing Co., Ltd.), 270, 3-chome Totsuka-cho, Shinjuku-ku, Tokyo 160, Japan.

* C. R. DePrima California Institute of Technology, Pasadena, CA 91109, will replace J. Dugundji until August 1974.

Copyright (C) 1973 by

Pacific Journal of Mathematics

All Rights Reserved 


\section{Pacific Journal of Mathematics}

\section{Vol. 49, No. $2 \quad$ June, 1973}

Wm. R. Allaway, On finding the distribution function for an orthogonal polynomial

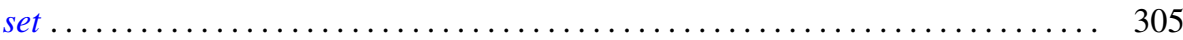

Eric Amar, Sur un théorème de Mooney relatif aux fonctions analytiques bornées... . 311

Robert Morgan Brooks, Analytic structure in the spectrum of a natural system . . . . 315

Bahattin Cengiz, On extremely regular function spaces . . . . . . . . . . . . . . 335

Kwang-nan Chow and Moses Glasner, Atoms on the Royden boundary . . . . . . . . . 339

Paul Frazier Duvall, Jr. and Jim Maxwell, Tame $Z^{2}$-actions on $E^{n} \ldots \ldots \ldots \ldots \ldots . .349$

Allen Roy Freedman, On the additivity theorem for $n$-dimensional asymptotic

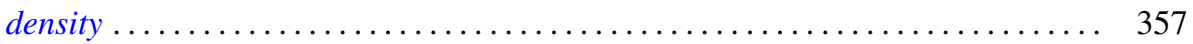

John Griffin and Kelly Denis McKennon, Multipliers and the group $L_{p}$-algebras . . . 365

Charles Lemuel Hagopian, Characterizations of $\lambda$ connected plane continua ....... 371

Jon Craig Helton, Bounds for products of interval functions . . . . . . . . . . . 377

Ikuko Kayashima, On relations between Nörlund and Riesz means . . . . . . . . . . 391

Everett Lee Lady, Slender rings and modules . . . . . . . . . . . . . . . . . . 397

Shozo Matsuura, On the Lu Qi-Keng conjecture and the Bergman representative

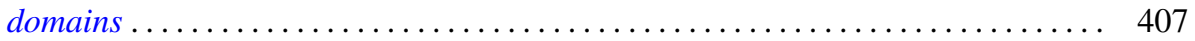

Stephen H. McCleary, The lattice-ordered group of automorphisms of an $\alpha$-set . . . 417

Stephen H. McCleary, o-2-transitive ordered permutation groups .......... 425

Stephen H. McCleary, o-primitive ordered permutation groups. II . . . . . . . . . 431

Richard Rochberg, Almost isometries of Banach spaces and moduli of planar

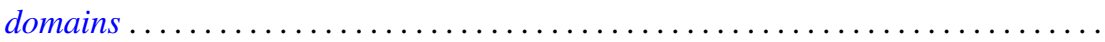

R. F. Rossa, Radical properties involving one-sided ideals . . . . . . . . . . . . . 467

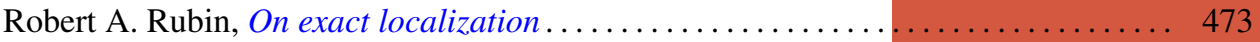

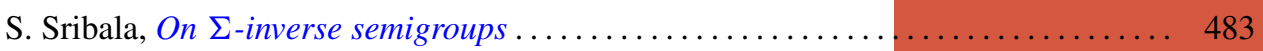

H. M. (Hari Mohan) Srivastava, On the Konhauser sets of biorthogonal polynomials suggested by the Laguerre polynomials ...................... 489

Stuart A. Steinberg, Rings of quotients of rings without nilpotent elements . ...... 493

Daniel Mullane Sunday, The self-equivalences of an $H$-space . . ............ 507

W. J. Thron and Richard Hawks Warren, On the lattice of proximities of $\check{C} e c h$

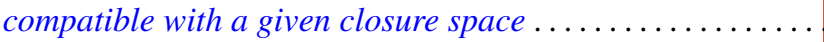

Frank Uhlig, The number of vectors jointly annihilated by two real quadratic forms determines the inertia of matrices in the associated pencil .

Frank Uhlig, On the maximal number of linearly independent real vectors annihilated simultaneously by two real quadratic forms ..............

Frank Uhlig, Definite and semidefinite matrices in a real symmetric matrix pencil . . 561

Arnold Lewis Villone, Self-adjoint extensions of symmetric differential operators . . . 569

Cary Webb, Tensor and direct products . ....................... 579

James Victor Whittaker, On normal subgroups of differentiable

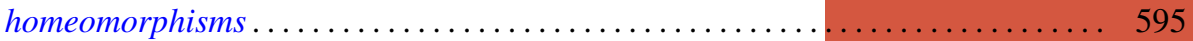

Jerome L. Paul, Addendum to: "Sequences of homeomorphisms which converge to

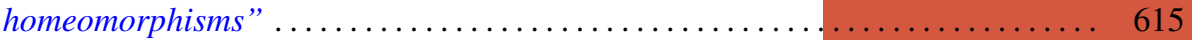

David E. Fields, Correction to: “Dimension theory in power series rings” ........ 616

Peter Michael Curran, Correction to: “Cohomology of finitely presented groups”. . . 617

Billy E. Rhoades, Correction to: “Commutants of some Hausdorff matrices” ...... 617

Charles W. Trigg, Corrections to: "Versum sequences in the binary system” ...... 619 